\title{
Review
}

\section{The foot in sport}

\author{
K P Sherman
}

\section{Introduction}

Foot disorders in sport can present very difficult diagnostic and treatment problems. Many treatments have not been subjected to properly conducted randomised controlled trials. Success in treating these disorders relies on a knowledge of the anatomy and kinesiology of the foot and an understanding of the interrelationship both between the different parts of the foot and between the foot and more proximal parts of the anatomy. Prevention and treatment require a knowledge of individual sports, and long term success requires a team approach which may involve coach, physiotherapist, doctor, orthotist, parent (in the case of children), and many other key personnel, but which, above all, must include the sportsperson him/herself.

In preparing this paper a search was made using Medline and the Cochrane database for the past 10 years, and a manual search of relevant journals was carried out.

\section{Orthopaedic}

Department, Castle Hill Hospital, Castle Road, Cottingham, East Yorkshire HU16 5JQ, United Kingdom

K P Sherman

Correspondence to: Mr K P Sherman.

Accepted for publication 9 September 1998
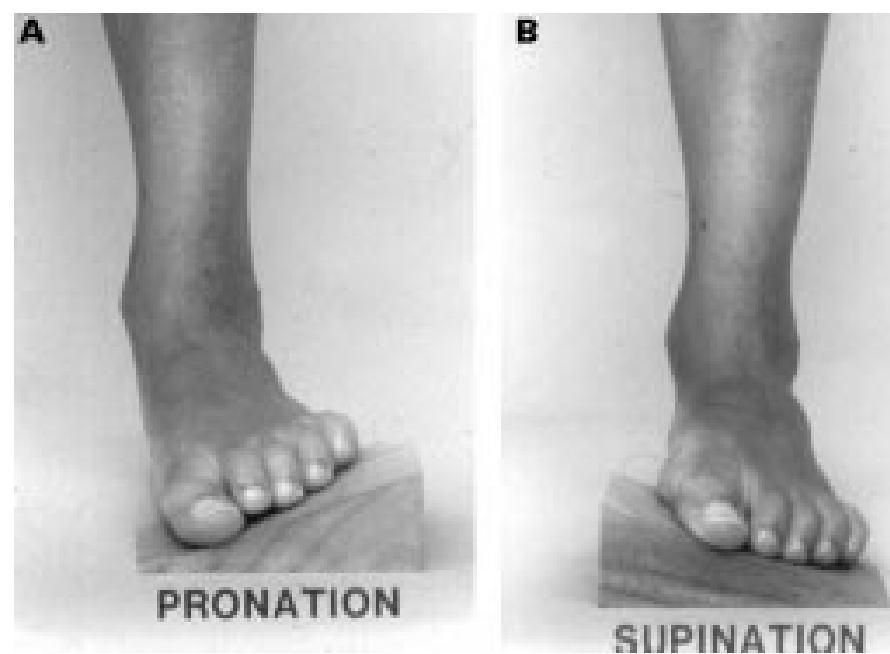

Figure 1 Photograph showing (A) pronated forefoot and (B) supinated forefoot. shape is produced partly by the "windlass effect"; the plantar aponeurosis inserts into the base of the proximal toe phalanges and when the toes are dorsiflexed the plantar fascia is tightened and the medial longitudinal arch increases in height. The axis of the subtalar joint lies about $41^{\circ}$ to the horizontal in the sagittal plane and $23^{\circ}$ to the midline axis of the foot in the transverse plane ${ }^{1}$; as a result of this oblique axis, torque around the longitudinal axis of the lower leg is converted into torque around the longitudinal axis of the foot (the "torque translator effect"). During normal gait the tibia internally rotates at initial foot loading and the foot pronates (see below) to allow flexibility and adaptation to the ground. At the start of "toe-off" the tibia externally rotates and the foot supinates to form a more rigid "lever". In addition to passive changes in shape, the longitudinal arches of the foot are influenced by muscle activity during propulsion, particularly the posterior and anterior tibialis and peroneus longus muscles. The intrinsic muscles attach proximally to the tarsal joints and during propulsion have an additional stabilising effect on the arches.

Examination of the foot must take these differing functions and postures into account.

\section{Terminology}

There is unfortunately a great deal of confusion in the terminology of foot movement and posture. It is important to distinguish between a description of a movement of a specific joint and the movement of the entire foot/ankle complex.

\section{INVERSION AND EVERSION}

These terms refer to tilting of the sole of the plantar aspect of the foot towards, or away from, the midline of the body respectively. The presence of a fixed inversion is often referred to as varus, and the presence of a fixed eversion is referred to as valgus. When applied to an individual joint varus and valgus also refer to angulation towards or away from the midline of the body respectively.

\section{ADDUCTION AND ABDUCTION}

Adduction and abduction refer to angulation towards or away from the midline, and in the mid- and fore-foot this movement is in a plane parallel to the plantar surface of the foot. In the case of the digits, the reference line is the midline of the second toe. 
PRONATION AND SUPINATION

These terms cause some of the greatest confusion. Gray's anatomy uses the analogy of a "twisted plate" when applied to the forefoot. If the heel is abducted on a level surface the midand fore-foot "untwist" (supinate) and the medial longitudinal arch is lowered. A similar untwisting or supination occurs if the heel is perpendicular to the ground and the forefoot is placed on a wedge based medially (fig 1). If the heel is adducted, the forefoot is pronated to maintain a level forefoot and the medial longitudinal arch is raised. Pronation and supination of the forefoot in this manner occurs around the axis of the least mobile second metatarsal. Pronation allows a downward rotation of the medial border of the foot and hallux towards the ground. Supination brings the lateral border into more direct plantigrade contact.

Clinically Alexander ${ }^{3}$ has described supination of the foot/ankle complex as a combination of adduction of the foot, inversion of the subtalar joint, and plantar flexion, and pronation as the converse (abduction, eversion, and dorsiflexion respectively). The terms pronation and supination are applied when the foot is load-bearing.

Pronation is not synonymous with pes planus

\section{History taking}

The history must include reference to a number of key features:

(a) acute or chronic onset of symptoms

(b) change of training (including any change in playing surface ${ }^{4}$ )

(c) change of footwear

(d) "start up pain" first thing in the morning or after sitting

(e) pain associated with particular exercises or activities

$(f)$ progressively increasing pain

(g) rest pain

(h) stiffness

(i) observable swelling

(j) weakness or instability

(k) callus formation

(l) any observed change of shape in the foot, or altered foot posture.

\section{Examination of the foot}

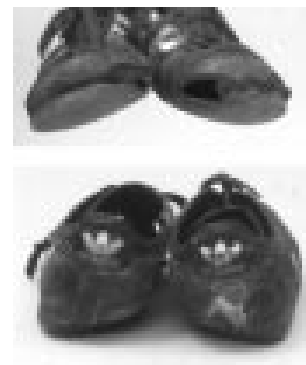

Examination of the foot must include evaluation of both static and dynamic functions and must be accompanied by an examination of the entire lower limb. Fixed deformities must be distinguished from flexible or passively correctable ones. The examination should include:

(a) standing (visualised from the front and from behind)

(b) standing with heel raised

(c) walking

(d) sitting

(e) prone with foot over end of couch
Figure 2 Severely worn sports shoes. (f) observation during performance of sport (either directly or by video-recording)

(g) entire lower limb

(h) footwear

With the patient standing, the shape and overall alignment of the foot can be assessed and the longitudinal arch observed. The patient should then stand on tiptoe (the heel raise test). This test should be performed with both feet together (to assess and compare heel alignment and longitudinal arches) and one foot at a time (the single heel raise test) to assess the Achilles tendon and tibialis posterior function. During the single heel raise test the knee must be kept extended to prevent the heel being lifted by rolling forward on the foot.

The examination performed with the patient sitting must be methodical and anatomically based. The foot should be examined for callosities or other evidence of skin or nail pathology. The positioning of callosities is an important clue to excessive loading of particular parts of the foot. The individual structures should be palpated to identify any swelling and the precise position of any tenderness. Examination of the joints of the forefoot and toes should be carried out with the ankle in neutral, dorsiflexed, and plantarflexed positions.

When the foot is examined with the patient prone, the forefoot should be adjusted in position until the medial border of the talar head aligns with the navicular tuberosity; in this position the calcaneus is in neutral position in the frontal plane. ${ }^{3}$ The forefoot frontal plane alignment can then be assessed relative to the calcaneus.

Owing to the interrelationship between foot shape and torsional and angular deformities in the tibia, knee, femur, and hip, the entire lower limb must be examined. Torsional and angular deformities that might normally be considered "within the normal range" may be of significance to the regular or high level sport participant, particularly if combined with other "mild" problems in the foot itself.

Box 1: Interrelationship between the joints and bones of the lower limb

- Valgus and varus of the knee will affect abduction/adduction of the heel when standing, and this will affect pronation/ supination of the fore-/mid-foot

- Owing to the torque translator effect of the subtalar joint, external rotation of the lower leg, with the foot on the ground, will be associated with a raised medial longitudinal arch (pronated forefoot); conversely internal rotation will lower the medial longitudinal arch

Finally attention should always be given to the footwear. Not only may inspection of the footwear give important clues to abnormal gait, or loading patterns, the footwear itself may be the source of the problem (fig 2). Inappropriate footwear for the particular sport, or for a 
particular foot, may contribute to, or cause, problems in the foot (and elsewhere in the lower limb)..$^{5-7}$ The "ideal" running shoe is unlikely to be optimal for court games such as squash because of a number of factors including the greater degree of twisting and lateral movement employed during court games. Appropriate footwear for use on artificial surfaces differs from that for use on "natural" surfaces because of different levels of friction.

\section{Disorders of the tendons}

Any of the tendons of the foot may be the source of symptoms. Pain arising from inflamed tendons typically takes the form of "start up pain", which initially settles after warming up but increases again during prolonged activity. Tenderness arising from tendons is characteristically worse when the corresponding muscle is contracted against resistance and on passive stretching of the tendon. In some areas (such as the dorsum of the foot), the diagnosis can usually be made clinically, but in other areas the diagnosis may be confused with other disorders such as chronic ligament strains. Magnetic resonance imaging (MRI) can be particularly useful in identifying the affected structure and pathology. ${ }^{8}$

Tendons may be affected by a number of different pathologies:

- Tenosynovitis

- Paratendinitis

- Tendinosis

- Acute tears

- Chronic tears

- Subluxation

POSTERIOR TIBIAL TENDON DISORDERS

Box 2: Posterior tibial malfunction

Examination findings in posterior tibial malfunction may include:

- Visible swelling distal to medial malleolus

- Valgus alignment of calcaneum when standing

- Reduced heel inversion when standing tiptoe

- Reduced ability to heel raise (single heel raise test) 9-10 times

- Reduced power of inversion of foot

- Reduced dorsiflexion of ankle

- Reduced passive inversion

- Palpable thickening and tenderness over tendon

Early problems may present with medial ankle pain when footwear is worn but more severe cases may develop valgus heel deformity collapse of the medial longitudinal arch, and an inability to run. Hallux valgus can be a contributing factor.

Acute tenosynovitis

This is more common in athletic persons and is frequently caused by overtraining. ${ }^{9} 10$ By contrast, chronic tenosynovitis is more common in the non-athletic and is often associated with hindfoot structural abnormalities.

Early acute tenosynovitis can be treated by reduction of activity, but more advanced cases require rest, ice, anti-inflammatory medication, and stretching followed by strengthening exercises (once the pain has subsided). If there is no response, a cast may be required. Surgery, consisting of debridement of synovial tissue, is sometimes needed; if extended proximal to the medial malleolus, a bridge of sheath should be left posterior to the medial malleolus to prevent subluxation.

\section{Chronic tenosynovitis}

This can often be treated with an orthotic (varus heel wedge and medial forefoot post) or a total contact insole (TCI), ${ }^{11}$ sometimes with a medial flare on the shoe, but may require surgical decompression or reconstruction with flexor digitorum longus.

\section{Acute rupture}

Acute rupture is rare before the age of $30 .{ }^{12}$ In complete rupture a palpable defect may be felt between the medial malleolus and the navicular, and there will be an absence of active inversion of the subtalar joint. Loss of posterior tibialis function causes the gastrosoleus to act at the talonavicular joint, eventually leading to stretching of the calcaneonavicular ligament and flat foot deformity. ${ }^{13-15}$ Complete rupture requires surgical treatment, usually using flexor digitorum longus to supplement the direct tendon repair.

Chronic tears

These are longitudinally orientated ${ }^{16}$ but may eventually lead to complete rupture. Conti ${ }^{17}$ has described a classification based on MRI findings.

\section{Dislocation of the posterior tibial tendon ${ }^{18}$}

This is very rare and conservative management is not successful. Surgical treatment is required involving relocation of the tendons, repair or reconstruction of the retinaculum, and deepening of the retromalleolar groove if necessary.

\section{Accessory navicular pain ${ }^{19}$}

There is no agreement on the cause of this pain. One theory is that the pain arises from microtrauma to the cartilage between the accessory bone and the navicular. ${ }^{20}$ Others think the cause is direct pressure on the prominent bone. Mild cases may be treated with a medial heel posting orthotic, moderate cases may be treated by casting for six weeks, but more persistent pain may require excision of those accessory navicular bones that are large and separated from the navicular by a cartilage layer ("Type II"). ${ }^{21}$ 
PERONEAL TENDON INJURIES

Box 3: Peroneal tendon injuries

- May involve peroneus longus, peroneus brevis or both

- May be acute or chronic

- May be primary or secondary to other pathology (such as ankle instability)

- May be asymptomatic and not require treatment

Tendinitis of peroneus brevis

This frequently occurs when sport is resumed after a lay off. Visible swelling, together with tenderness, may be seen along the line of the tendon sheath distal to the lateral malleolus. ${ }^{22}$ The condition usually responds to rest, ice, compression and elevation (RICE) and antiinflammatory medication. In chronic tendinitis there may be little swelling or tenderness and the condition may be confused with lateral ankle ligament problems. In difficult cases, MRI scanning may be helpful, showing fluid within the tendon sheath. ${ }^{23}$ Treatment of the chronic condition may include stretching and strengthening exercises, taping and bracing, or the use of a TCI with lateral heel wedge and, in resistant cases, surgical debridement. As chronic tendinitis may lead to longitudinal tearing, injection of corticosteroid for chronic inflammation may be hazardous. ${ }^{24}$

Longitudinal tears of the peroneus brevis tendon These may occur after overuse, particularly in runners or walkers. They may also be associated with ankle instability and can give rise to pain that is greater than would normally be expected with chronic instability. ${ }^{25}$ Subluxation of peroneus brevis may also lead to longitudinal tears. MRI can be particularly useful in confirming the diagnosis. Tears commence at the tip of the lateral malleolar groove. Conservative treatment with stretching exercises and the use of bracing or high top shoes to prevent excessive inversion will be successful in many cases, but for resistant cases surgical debridement with repair of the tear (which is usually found on the deep surface of the tendon) may be required. Any contributing condition (such as ankle instability, tendon subluxation, impinging bone) should be dealt with simultaneously.

Complete rupture of the peroneus brevis tendon This requires surgical repair. In late-presenting complete tears, where retraction has occurred, the tendon may be sutured to the lateral calcaneus, or a plantaris tendon graft used. ${ }^{26}$

Acute inflammation of the peroneus longus tendon Typically coming on when sport is resumed after a lay off, this often causes pain over the lateral heel on raising the heel off the ground when running or turning. Treatment is similar to that for peroneus brevis tendinitis. An orthotic to prevent midfoot pronation may also help.
Acute rupture of the peroneus longus tendon This occurs where the tendon turns under the cuboid. Where an os peroneum is present ( $20 \%$ of the population), it occurs through the accessory bone and may then be diagnosed by $x$ ray analysis, which shows a proximal migration of the os peroneum. When no os peroneum is present, MRI scanning may be needed to confirm the diagnosis. ${ }^{27}$ The acute rupture is sometimes preceded by several weeks of symptoms. Conservative treatment can be successful in mildly symptomatic cases but operative suture (sometimes with a tendon graft) is often required.

Chronic tears of the peroneus longus tendon

These tears occur longitudinally and are more common in middle aged athletes. The tears usually commence where the tendon turns under the cuboid and present with pain in the lateral border of the foot. There may be swelling over the lateral cuboid. An os peroneum, when present, may appear fragmented in radiographs. Conservative measures, including stretching and strengthening exercises and an orthotic to control excessive pronation of the foot, may be successful. Surgical treatment may include debridement, tenosynovectomy, advancement and repair of the tendon (with or without tendon grafting) when complete rupture has occurred, or suturing to the lateral calcaneus when the tendon has ruptured and retracted and has become fixed.

\section{Dislocating peroneal tendons}

This condition is more common in the presence of a shallow groove posterior to the fibula, pes planus, hindfoot valgus, or recurrent ankle sprains. Radiology sometimes reveals an avulsed fragment from the posterolateral border of the lateral malleolus. A number of operations have been described for the treatment of symptomatic dislocation. ${ }^{24}{ }^{28}{ }^{29}$ Asymptomatic dislocation does not require treatment.

Care must be taken to avoid damage to the sural nerve when treating peroneal injuries surgically.

OTHER TENDONS

Other tendons in the foot may also develop acute or chronic inflammatory problems or rupture.

Box 4: Tendon problems

- Typically have "start up pain"

- Swelling of tendon often seen in acute cases but less common in chronic problems

- Tenderness usually exacerbated by resisted contraction of relevant muscle

- Tenderness usually exacerbated by passive stretching of tendon 


\section{Disorders of the nerves}

Box 5: Nerve lesions
ACUTE
- Contusions
- Lacerations in association with other
injuries
- Acute traction injury-in association
with other injuries
CHRONIC
- Recurrent traction
- Recurrent compression

In chronic nerve lesions, symptoms may only be present during exercise.

INTERDIGITAL NEUROMA

This is characterised by pain which occurs mainly when footwear is being worn. Burning, shooting, or tingling pain radiates into the toe or toes. Stretching of the nerve may result from excessive repeated dorsiflexion of the toes. Swelling of the metatarsophalangeal joint may also impinge against the nerve. Neuromata occur most commonly in the third/fourth web space, and next most commonly in the second/ third web space. The clinical test is to squeeze the forefoot at the level of the metatarsal heads, dorsiflex the toes, and palpate on the plantar surface of the web space. A search should also be made for more proximal tenderness as this condition can be confused with nerve compression in the tarsal tunnel or more proximally. Treatment with a metatarsal pad placed proximally to the metatarsal heads is often useful. Corticosteroid injections can be given. Surgical excision of the neuroma is often required.

TARSAL TUNNEL SYNDROME ${ }^{30} 31$

The tarsal tunnel extends from proximal to the malleolus into the foot and is roofed by the flexor retinaculum. In the retromalleolar region, the tibial nerve divides into the medial plantar nerve, the lateral plantar nerve, the first branch of the lateral plantar nerve, and the calcaneal nerve; in the distal part of the tarsal tunnel these nerves run in separate tunnels. ${ }^{32}$ The possibility of nerve entrapment more proximally should be considered. Tarsal tunnel syndrome may occur spontaneously or secondary to swelling of other structures within, or adjacent to, the tarsal tunnel (such as ganglia or flexor tendon tenosynovitis). Spontaneous cases are often associated with a foot that is repeatedly pronated such as the inside foot in a track runner. The typical symptoms are burning or shooting pains extending from the medial ankle into the foot, often with paraesthesia. ${ }^{33}$ Electrophysiological studies may help in diagnosis. Conservative treatments include the use of a medial heel and sole wedge to prevent excessive pronation, taping or bracing, and anti-inflammatory medication. In resistant cases, surgical decompression of the nerve with division of the medial retinaculum, release of the deep fascia of the abductor, and removal of any impinging pathology is performed. Nerve entrapment within the indi- vidual distal tarsal tunnels may occur either in isolation or in combination with proximal tarsal tunnel compression. ${ }^{34}$ Entrapment of the medial calcaneal branches of the posterior tibial nerve may cause heel pain and can occur in association with other causes of subcalcaneal pain.

MEDIAL PLANTAR NERVE ENTRAPMENT

This nerve may become entrapped by the fascia beneath the navicula and talus or by the abductor hallucis. ${ }^{35}$ The pain will usually be burning or shooting and radiate towards the plantar aspect of the medial two toes. The pain may be accompanied by tingling or numbness. High arch supports can exacerbate the symptoms. A positive Tinel's sign is helpful in making a diagnosis. Tenderness may occur along the medial arch. The condition may be confused with flexor tenosynovitis, but unlike in the latter condition the tenderness is not made worse by resisted flexion of the toes, nor by passive dorsiflexion of the toes. High arches should be removed, and conservative measures including cortisone injection adjacent to but not into the nerve may be successful. In resistant cases, surgical decompression of the neurovascular bundle is performed at the site of maximal tenderness.

ENTRAPMENT OF THE FIRST BRANCH OF THE LATERAL PLANTAR NERVE

This nerve usually separates from the lateral plantar nerve behind the medial malleolus. The nerve may become trapped where it passes between abductor hallucis and quadratus plantae. This condition often presents with medial plantar heel pain, which in some cases radiates into the lateral foot or proximally, and in $10-15 \%$ of cases is associated with plantar fasciitis. ${ }^{36}$ On clinical examination, maximum tenderness is found along the abductor muscle on the medial aspect of the heel. Neuritic pain or paraesthesia may occasionally be produced by such palpation. ${ }^{5}$ There is no consensus on the usefulness of neurophysiology in this condition. ${ }^{37}$ Treatment can be commenced with heel pads, a heel lift, stretching exercises for the Achilles tendon and plantar fascia, and anti-inflammatory medication. Steroid injections adjacent to the nerve can be used. When surgical treatment is undertaken, the superficial and deep abductor fascia is released together with part of the medial plantar fascia. Surgery is not always successful in relieving the symptoms. ${ }^{36} 38$

PERONEAL NERVE ENTRAPMENT

The deep peroneal nerve can be trapped or impinged upon at various sites. Entrapment may be under the extensor hallucis brevis or the retinaculum or may be due to underlying osteophytes with tight footwear. The pain is vague and felt over the dorsum of the foot. There is occasionally transient numbness in the first web space. The presence of weakness of the extensor digitorum brevis or the toe extensors indicates a lesion proximal to the ankle. Treatment consists of avoidance of compression (footwear modifications etc). Surgical 
removal of osteophytes or bony ridges is occasionally required.

The superficial peroneal nerve is usually trapped proximal to the ankle where the nerve pierces the deep fascia; a search should be made for tenderness about $10 \mathrm{~cm}$ proximal to the lateral malleolus in cases where pain is felt over the dorsum of the foot. ${ }^{39}$ Styf $^{40}$ has described three tests for this condition.

SURAL NERVE ENTRAPMENT OR DAMAGE

Symptoms may arise from this nerve as a result of compression by fibrous tissue following recurrent ankle sprains, or ganglia etc. The nerve or its branches is also particularly vulnerable during lateral approaches to the ankle or hindfoot.

\section{Box 6: Nerve entrapment syndromes}

- Should be considered in cases of resistant pain in the foot

- May easily be confused with ligament or tendon problems

- Typically, but not always, present with burning or shooting pain, often of vague distribution

- May or may not be accompanied by tingling or numbness

- Symptoms may only be present during exercise

- The most important diagnostic tool is careful palpation along the anatomical course of individual nerves

- In all cases a careful search should be made for proximal entrapment or nerve pathology

\section{Disorders of the joints}

BUNIONS

Painful bunions resulting from hallux valgus can be treated with an insole with a good medial arch support to reduce excessive pronation. When the first metatarsal is shorter than the second ("Morton's foot"), an orthosis with an extension under the first metatarsal head can be used ("Morton's extension"). In more advanced cases surgery may be required, usually a first metatarsal osteotomy; the method chosen should minimise shortening of the first metatarsal. ${ }^{41}{ }^{42}$ The aim is to restore congruity of the first metatarsophalangeal joint. ${ }^{43}$

\section{HALLUX RIGIDUS}

The early stages of this condition often occur in the late teens but symptoms often do not start until dorsiflexion of the hallux becomes significantly restricted (normal dorsiflexion between axes of first metatarsal and proximal phalanx is $90^{\circ}$ ). An orthosis giving more rigidity to the sole may help symptoms, ${ }^{44}$ or a rocker may be fitted to the sole of the shoe.

TURF TOE

This condition is essentially a severe sprain of the first metatarsophalangeal joint, usually from hyperextension. It is more common when light flexible shoes are used on artificial playing surfaces. ${ }^{45}$ Firmer soled shoes or firmer insoles should be used by those with a reduced range of dorsiflexion of the first metatarsophalangeal joint. ${ }^{46}$ The joint appears swollen and tender, with increased pain on passive dorsiflexion. Initial treatment with RICE should be followed by physiotherapy and taping. ${ }^{66}$ Return to sport should be avoided until a near full pain free range of motion has been restored, to prevent prolonged disability. ${ }^{11}$

FREIBERG'S DISEASE ${ }^{47} 48$

This osteochondrosis of the metatarsal head is more common in female adolescents (about 5:1 female to male). The cause has not been established with certainty but is probably multifactorial. ${ }^{49}$ The second metatarsal head is the most frequently involved but it can occur in any of the metatarsals. Presenting symptoms can be pain, restriction of movement in the metatarsophalangeal joint, and sometimes swelling. In some cases symptoms in the initial stages may be transient or not occur at all. Late cases present with osteoarthritis of the metatarsophalangeal joint. Radiological changes are variable and do not correlate with prognosis, ${ }^{50}$ and in the earliest stage there may be no radiological changes; a bone scan can help to identify such cases. There is no consensus on the most effective conservative or surgical treatments. Reduction of activities and casting has been frequently advised, together with an orthotic to relieve loads on the affected joint. Injections and various forms of physiotherapy have also been advocated. Of the various operations described, dorsal wedge osteotomy of the metatarsal neck and shortening of the metatarsal are among the least radical. ${ }^{51}$

\section{TARSAL COALITION}

Coalitions most commonly occur in the subtalar or calcaneonavicular joints. The loss of hindfoot mobility leads to abnormally high stresses on other structures in the foot. Subtalar/hindfoot mobility should always be examined when investigating foot problems. Bony coalitions may be seen in plain radiographs, but are often more easily demonstrated with computed tomography. MRI will show both bony and fibrous coalitions.

\section{Disorders of the bones}

\section{STRESS FRACTURES}

Characteristically the pain occurs at a progressively earlier stage of exercise as the condition develops. ${ }^{52}$ Various bones may be affected including metatarsals and calcaneum..$^{53}$ Stress fractures of the metatarsals may occur secondary to hallux valgus. In the early stages these fractures may not be visible in plain $x$ ray films; in these cases technetium bone scans or MRI scans are useful. ${ }^{8}$

\section{SEVER'S DISEASE (APOPHYSITIS OF THE} CALCANEAL APOPHYSIS $)^{54}$

This condition usually occurs in the younger age group (8-12 years) and presents with pain over the posterior aspect of the heel. The condition is self limiting but may require a reduction in activities for symptomatic relief. 
HEEL SPURS

Despite their association with painful heel conditions,there is a lack of evidence that heel spurs actually cause pain. ${ }^{36556}$ Although heel spurs are more common in relatively young people with heel pain than in those without, many spurs are asymptomatic. ${ }^{57}$

The presence of heel spurs in patients with heel pain does not necessarily indicate a causal relationship. Most heel spurs do not require removal for resolution of symptoms.

\section{Other disorders of the soft tissues}

METATARSALGIA

Pain under the metatarsal heads may result from splaying of the forefoot, as may occur in hallux valgus or excessive pronation of the forefoot. Treatment with padding proximal to the metatarsal heads, sometimes with taping of the forefoot, will often relieve the symptoms.

PLANTAR FASCIITIS

Often erroneously used to describe any pain on the plantar surface of the heel, this condition is estimated to affect $10 \%$ of runners. ${ }^{58}$ The plantar fascia extends from the calcaneum to the base of the proximal phalanges. The stress on the fascia may be increased by hindfoot valgus, weak peroneal muscles, or reduced first metatarsal mobility. Pes planus, pes cavus, and restricted subtalar movement may all be predisposing factors. ${ }^{5960}$ The pain is usually located at the origin of the fascia from the medial aspect of the plantar surface of the heel, but may also occur in the mid part of the longitudinal arch. Pain may be increased by dorsiflexing the toes, and can occur when heel walking or on tiptoe. It must be distinguished from entrapment of the first branch of the lateral plantar nerve and from other rarer causes of heel pain such as bone cysts in the calcaneum and arthritis of the subtalar joint. This condition usually responds to conservative management, which may include corrective heel orthotics ${ }^{61}$ (usually to reduce pronation), heel pads, taping, intrinsic foot exercises, ultrasound, ${ }^{62}$ strengthening and stretching exercises, hydrocortisone injections, and nonsteroidal medication. ${ }^{56}$ Night splints to prevent nocturnal contracture of the plantar fascia and Achilles tendon have been described. ${ }^{63} \mathrm{~A}$ search for the cause should be made, with appropriate adjustments to the training programme. ${ }^{6465}$ A large number of different operations and surgical approaches have been described for the small proportion of cases $(<5 \%)$ that do not respond to conservative measures. The most common is a plantar fasciotomy, which can be carried out by an open procedure or endoscopically. ${ }^{66}$ Care must be taken not to damage the medial calcaneal nerves.

HEEL PAD PAIN

The heel fat pad has a highly specialised structure, with fat globules enclosed between fibroelastic septa. ${ }^{67}$ Pain arising in the fat pad presents with similar pain to plantar fasciitis under the heel ${ }^{69}$ and results from direct trauma to the heel pad. Loss of subtalar joint movement prevents pronation of the foot at heel strike and may predispose to heel pad pain. There should be no pain when walking on tiptoe. Treatment should include shock absorbing heel pads and appropriate footwear advice.

Karr $^{59}$ describes an extensive differential diagnosis of subcalcaneal pain. Once inflammation has developed, secondary involvement of other structures may occur. ${ }^{54}$ The aetiology of heel pain in athletes is usually multifactorial.

Box 7: Summary of the more common causes of subcalcaneal pain

- Plantar fasciitis

- Heel pad syndrome

- Entrapment of the first branch of the lateral plantar nerve

- Entrapment of the calcaneal branches of the posterior tibial nerve

- Stress fracture of the calcaneum

- Inflammatory arthritis-for example, seronegative spondyloarthropathies ${ }^{70}$

\section{Conclusion}

The management of foot symptoms in sport can be challenging. A careful history and examination, together with an understanding of the anatomy and kinesiology of the foot, and its relation to the rest of the lower limb, together with judicious use of appropriate special investigations, will in most cases enable an accurate diagnosis to be made and a logical treatment programme to be planned. A search should always be made for preventable factors in order to avoid recurrence of the problem.

1 Simon SR, Alaranta H, An KN, et al. In: Simon SR, ed. Orthopaedic basic science. American Academy of OrthopaeOrthopaedic basic science. Ame
dic Surgeons, 1994:592-600.

2 Warwick R, Williams PL (eds). Gray's anatomy. Edinburgh: Longman.

3 Alexander IJ. The foot: examination and diagnosis. Edinburgh: Churchill-Livingstone, 1990

4 Nigg BM, Segesser B. The influence of playing surfaces on the load on the locomotor system and on football and tennis injuries. Sports Med 1988;5:375-85.

5 Bird S, Black N, Newton P. Sports injuries: causes, diagnosis, treatment and prevention. Cheltenham: Stanley Thornes (Publishers) Ltd, 1997.

6 Norris CM. Sports injuries, diagnosis and management, 2nd ed. Oxford: Butterworth-Heinemann, 1998.

7 Robbins SE, Gouw GJ. Athletic footwear and chronic overloading. Sports Med 1990;9:76-85.

8 Roberts MD, Pomeranz SJ. Current status of magnetic Roberts $\mathrm{MD}$, Pomeranz $\mathrm{SJ}$. Current status of magnetic
resonance in radiologic diagnosis of foot and ankle injuries. resonance in radiologic diagnosis of fo
Orthop Clin North Am 1994;25:61-8.

9 Outerbridge AR, Mecheli LJ. Overuse injuries in young athOuterbridge AR, Mecheli LJ. Overuse in
letes. Clin Sports Med 1995;14:503-16.

10 Teitz C, Garrett W, Miniaci A, et al. Tendon injuries in athTeitz C, Garrett W, Miniaci A, et al. Tendon injuries in
letic individuals. Instruct Course Lect 1997;46:575-82.

11 Mascaro TB, Swanson LE. Rehabilitation of the foot and ankle. Orthop Clin North Am 1994;25:147-59.

2 Woods L, Leach RE. Posterior tibial tendon rupture in athletic people. Am $\mathcal{F}$ Sports Med 1991;19:495-8.

3 Basmajian JV, Stecko G. The role of muscles in arch support of the foot. I Bone foint Surg [Am] 1963;45:1184-90.

14 Funk DA, Cass JR, Johnson KA. Acquired adult flat foot secondary to posterior tibial tendon pathology. $\mathcal{F}$ Bone foint Surg $[A m]$ 1986;68:95-102.

15 Mann RA, Thompson FM. Rupture of the posterior tibial tendon causing flat foot: surgical treatment. F Bone foint Surg $[A m]$ 1985;67:556-61.

16 Conti SF. Posterior tibial tendon problems in athletes. Orthop Cin North Am 1994;25:109-21.

17 Conti SF, Michelson J, Jahss MH. Clinical significance of magnetic resonance imaging in preoperative planning for reconstruction of posterior tibial tendon ruptures. Foot Ankle 1992;13:208-14. 
18 Ouzounian TJ, Myerson MS. Dislocation of the posterior tibial tendon. Foot Ankle 1992;13;215-19.

19 Mygind HB. The accessory tarsal scaphoid: clinical features and treatment. Acta Orthop Scand 1953;23:142-51.

20 Grogan DP, Gasser SI, Ogden JA. The painful accessory navicular: a clinical and histopathological study. Foot Ankle 1989;10:164-9.

21 MacNicol MF, Voutsinas S. Surgical treatment of the symptomatic accessory navicular. F Bone foint Surg $[\mathrm{Br}]$ 1984;66:218-26.

22 Parvin RW, Fort LT. Stenosing tenosynovitis of the common peroneal tendon sheath. $\mathcal{F}$ Bone foint Surg [Am] 1956;38: 1352-7.

23 Rosenberg ZS, Cheung Y, Jahss MH. Computer tomography scan and magnetic resonance imaging of ankle tendons. An overview. Foot Ankle 1988;8:297-307.

24 Sammarco GJ. Peroneal tendon injuries. Orthop Clin North Am 1994;25:135-45.

25 Shoda E, Kurosaka M, Yoshiya S, et al. Longitudinal ruptures of the peroneal tendons. A report of a rugby ruptures of the peroneal tendons. A

26 Stark HH, Anderson DR, Zemel NP, et al. Bridge flexor tendon grafts. Clin Orthop 1989;242:51-9.

27 Tehranzadeh J, Stoll DA Gabriele OM Case report 271. Posterior migration of the os peroneum of the left foot, indicating a tear of the peroneal tendon. Skeletal Radiol 1984;12:44.

28 Jones E. Peroneal dislocation. F Bone foint Surg 1992;14: 574

29 Pozo JL, Jackson AM. A rerouting operation for dislocation of peroneal tendons: operative technique and case report. Foot Ankle 1984;5:42.

30 Jackson DL, Haglund B. Tarsal tunnel syndrome in athletes: case reports and literature review. Am $\mathcal{F}$ Sports Med 1991;19:61-5.

31 Schon LC. Nerve entrapment, neuropathy, and nerve dysfunction in athletes. Orthop Clin North Am 1994;25:4759.

32 Heimkes B, Posel P, Stotz S, et al. The proximal and distal tarsal tunnel syndromes: an anatomic study. Int Orthop tarsal tunnel

33 Edwards WE, Lincoln R, Bassett FH et al. The tarsal tunnel syndrome: diagnosis and treatment. $¥ A M A$ 1969;207:716.

34 Gessini L, Jandolo B, Peitrangel A. The anterior tarsal tunnel syndrome: report of four cases. F Bone foint Surg [Am] 1984;66:786

35 Murphy PC, Baxter DE. Nerve entrapment of the foot and ankle in runners. Clin Sports Med 1985;4:753

36 Baxter DE, Thigpen CM. Treatment of chronic heel pain by surgical release of the first branch of the lateral plantar nerve. Clin Orthop 1992;279:229-36.

37 Schon LC, Glennon TP, Baxter DE. Heel pain syndrome: electrodiagnostic support for nerve entrapment. Foot Ankle 1993;14:129-35.

38 Lutter LD. Surgical decisions in athlete's subcalcaneal pain. Am $\mathcal{F}$ Sports Med 1986;14:481-5.

39 Lowdon IMR. Superficial peroneal nerve entrapment: case report: $\mathcal{F}$ Bone foint Surg [Br] 1985;67:58

40 Styf J. Entrapment of the superficial peroneal nerve: diagnosis and results of decompression. F Bone foint Surg $[\mathrm{Br}]$

41 Johnson K, Cofield R, Morrey B. Chevron osteotomy for hallux valgus. Clin Orthop 1979;142:44-7.

42 Mitchell LA, Baxter DE. A Chevron-Akin double osteotomy for correction of hallux valgus. Foot Ankle 1991;12 $7-14$.

43 Baxter DE. Treatment of bunion deformity in the athlete. Orthop Clin North Am 1994;25:33-9.
44 Clanton TO, Butler JE, Eggert A. Injuries to the metatarsophalangeal joints in athletes. Foot Ankle 1986;7: metatarsoph

45 Doller J, Strothers S. Turf toe, an acute inflammatory response to athletic activity on artificial playing surfaces. $\mathcal{F}$ Am Podiatr Med Assoc 1978;68:512.

46 Bowers KD, Martin RB. Turf toe: a shoe-surface related football injury. Med Sci Sports Exerc 1976;8:81-3.

47 Freiberg A. Infraction of the second metatarsal bone: a typical injury. Surg Gynaecol Obstet 1914;19:191-3.

48 Katcherian DA. Treatment of Freiberg's disease. Orthop Clin North Am 1994;25:69-81.

49 Duthie R, Houghton G. Constitutional aspects of the osteochondroses. Clin Orthop 1981;158:19-27.

50 Omer G. Primary articular osteochondroses. Clin Orthop 1981;158:33-41

51 Kinnard P, Lirette R. Dorsiflexion osteotomy in Freiberg's disease. F Bone foint Surg [Br] 1991;73:864-5.

52 Coady CM, Micheli LJ. Stress fractures in the paediatric athlete: Clin Sports Med 1997;16:225-38.

53 Gillespie WJ. The prevention and management of lower limb stress fractures (Cochrane Review). In: The Cochrane library, issue 2. Oxford: Update Software, 1998.

54 Bernhardt DT, Landry GL. Sports injuries in young athletes. Adv Pediatr 1995;42:465-500.

55 Lapidus PW, Guidotti FP. Painful heel: report of 323 patients with 364 painful heels. Clin Orthop 1965;39:17986

56 Schepsis AA, Leach RE, Gorzyca J. Plantar fasciitis, etiology, treatment, surgical results and review of the literature. Clin Orthop 1991;266:185-96.

57 Tanz SS. Heel pain. Clin Orthop 1963;28:169-78.

8 Crawford F, Atkins D, Edwards J, et al. Treatments for painful plantar heel syndrome (Cochrane Review). In: The Cochrane library, issue 2. Oxford: Update Software, 1998.

59 Karr SD. Subcalcaneal heel pain. Orthop Clin North Am 1994;25:161-75.

60 Kibler WB, Goldberg C, Chandler TJ. Functional biomechanical deficits in running athletes with plantar fasciitis. Am 7 Sports Med 1991;19:66-71.

61 Janisse DJ. Indications and prescriptions for orthoses in sports. Orthop Clin North Am 1994;25:95-107.

62 Crawford F, Snaith $M$. How effective is therapeutic ultrasound as a treatment for plantar heel pain? Ann Rheum Dis 1996;55:265-7.

63 Wapner KL, Sharkey PF. The use of night splints for treatment of recalcitrant plantar fasciitis. Foot Ankle 1991;12: 135-8.

64 Warren BL. Plantar fasciitis in runners, treatment and prevention. Sports Med 1990;10:338-45.

65 Bordelon RL. Subcalcaneal pain: present status, evaluation and management. Instr Course Lect 1984;33:283-7.

66 Barrett SL, Day SV, Pignetti TT, et al. Endoscopic plantar fasciotomy: a multi-surgeon prospective analysis of 652 cases. Foot Ankle 1995;34:400-6.

67 Jahss $\mathrm{MH}$, Michelson JD, Desai P, et al. Investigations into the fat pads of the sole of the foot: anatomy and histology. Foot Ankle 1992;13:233-42.
For fat pads of the sole of the

68 Jahss MH, Kummer F, Michelson JD. Investigations into the fat pads of the sole of the foot: heel pressure studies. Foot Ankle 1992;13:227-32.

69 Katoh Y, Chao EY, Morrey BF, et al. Objective technique for evaluating painful heel syndrome and its treatment. Foot Ankle 1983;3:227-37.

70 Gerster JC, Piccinin P. Enthesiopathy of the heels in juvenile onset seronegative $\mathrm{B}-27$ positive spondyloarthropathy. $\mathcal{F}$ Rheumatol 1985;12:310-14. 